\title{
Dieta e comportamento alimentar de Malacoctenus delalandii (Perciformes: Labrisomidae)
}

\author{
Pedro Henrique Cipresso Pereira ${ }^{1,2}$ \& Giuliano Buzá Jacobucci ${ }^{1,2}$ \\ ${ }^{1}$ Laboratório de Ecologia, Instituto de Biologia, \\ Campus Umuarama, Universidade Federal de Uberlândia - UFU, \\ Rua Ceará, s/n, CEP 38400-902, Uberlândia, MG, Brasil \\ ${ }^{2}$ Autor para correspondência: Pedro Henrique Cipresso Pereira, e-mail: pedrohcp2@yahoo.com.br
}

PEREIRA, P.H.C. \& JACOBUCCI, G.B. Diet and feeding behavior of Malacoctenus delalandii (Perciformes: Labrisomidae). Biota Neotrop., 8(3): http://www.biotaneotropica.org.br/v8n3/en/ abstract?article+bn01808032008

\begin{abstract}
This work analyzed the diet composition and the feeding behavior of Malacoctenus delalandii, a carnivorous species, with diurnal habit, that lives in holes and crevices of rock areas in tropical waters. For diet evaluation, 31 individuals were collected during diving using hand nets, in the Fortaleza beach, Ubatuba. The feeding offer was analyzed comparing the stomach contents of the collected individuals with ten samples of the Amphiroa beauvoisii calcareous algal phytal, the species foraging site. To describe the feeding behavior of M. delalandii 44 observation sections were performed using ad libitum and focal animal methods. The food items considered constant were crustaceans belonging to the order Isopoda (75.8\%) and the suborder Gammaridea (86.2\%), confirming the species carnivorous feeding habit. There was a significant difference $(\mathrm{G}=278.89$, $\mathrm{df}=70, \mathrm{p}<0.01$ ) between the availability of items in the substrate and the occurrence in the stomachs. A total of 410 minutes ( 6.8 hours) of direct underwater observations were performed, and we concluded that the individuals use the grubber excavation while moving feeding strategy. The species presented a foraging rate of $0.74 \pm 0.164$ bites per minutes (average \pm standard deviation), evidencing low feeding activity and cryptobenthic habit.
\end{abstract}

Keywords: blenny, trophic ecology, stomach contents, foraging activity, southeast Brazil.

PEREIRA, P.H.C. \& JACOBUCCI, G.B. Dieta e comportamento alimentar de Malacoctenus delalandii (Perciformes: Labrisomidae). Biota Neotrop., 8(3): http://www.biotaneotropica.org.br/v8n3/pt/ abstract?article+bn01808032008

Resumo: O presente estudo teve como objetivo analisar a composição da dieta e o comportamento alimentar de Malacoctenus delalandii, uma espécie carnívora, de hábitos diurnos, que habita tocas e frestas de ambientes rochosos de águas tropicais. Para avaliação da dieta, foram coletados 31 indivíduos utilizando puçás, na praia da Fortaleza, município de Ubatuba. A oferta alimentar foi analisada comparando-se o conteúdo estomacal dos indivíduos com dez amostras do fital da alga calcária Amphiroa beauvoisii, local de forrageio da espécie. Para descrever o comportamento alimentar de $M$. delalandii foram realizadas 44 sessões de observação usando técnicas ad libitum e animal focal. Os itens alimentares considerados constantes foram os crustáceos pertencentes à ordem Isopoda $(75,8 \%)$ e à subordem Gammaridea $(86,2 \%)$, confirmando o hábito alimentar carnívoro da espécie. Houve uma diferença significativa $(\mathrm{G}=278,89$, g.L. $=70, \mathrm{p}<0,01)$ entre a disponibilidade de itens no substrato e a ocorrência destes nos estômagos. Foram realizados 410 minutos (6,8 horas) de observações subaquáticas diretas e concluiu-se que os indivíduos utilizam a estratégia alimentar de especulação do substrato ("grubber excavation while moving”). A espécie apresentou uma taxa de forrageamento de 0,74 \pm 0,164 mordicadas per minutes (média \pm desvio padrão), evidenciando baixa atividade alimentar e hábito criptobêntico.

Palavras-chave: blênio, ecologia trófica, conteúdos estomacais, atividade de forrageamento, sudeste do Brasil. 


\section{Introdução}

As regiões de costões rochosos constituem um dos ambientes marinhos mais densamente habitados e com maior diversidade de espécies animais e vegetais existindo, portanto, uma grande quantidade de alimento disponível para os peixes que habitam esses locais (Moring 1979).

Estudos sobre a biologia alimentar de peixes, com análises de conteúdo estomacal, podem gerar subsídios para um melhor entendimento das relações entre a ictiofauna e os demais organismos da comunidade aquática, consistindo em uma importante ferramenta na definição de estratégias para o manejo sustentável dos ecossistemas (Hahn \& Delariva 2003).

$\mathrm{O}$ estudo do regime alimentar de peixes marinhos tem grande importância, existindo uma relação bastante estreita entre quantidade, qualidade, disponibilidade, distribuição e abundância de alimento (Kawakami \& Amaral 1983). Além da relevância ecológica para a compreensão do funcionamento dos sistemas, a trofodinâmica tem importância prática no que tange ao uso sustentado do ambiente, auxiliando na compreensão de muitos processos biológicos e exploração racional de estoques de peixes e de outros organismos marinhos de valor econômico (Amaral \& Migotto 1980, Costa et al. 1987).

Peixes bentívoros apresentam uma grande diversidade de comportamentos alimentares (Gerking 1994), além de várias adaptações morfológicas relativas ao comportamento de forrageamento e fuga de predadores, e exploram de forma eficiente os substratos bentônicos consolidados e não consolidados (Hobson \& Chess 1986, McCornick 1995, Platell et al. 1998). Esses peixes possuem usualmente apêndices sensoriais e maxilares inferiores móveis, predando invertebrados bentônicos associados ao fundo, enterrando a boca no substrato e engolindo parte do sedimento (Hobson \& Chess 1986, Sazima 1986, Edgar \& Shaw 1995).

A escolha do alimento mais apropriado às necessidades nutricionais e adaptativas dos peixes depende da oferta alimentar no ambiente e da variação sazonal (Mittelbach \& Persson 1998). Desse modo, a presença de determinado tipo de alimento nos estômagos não significa, necessariamente, que se trata do item preferido, tendo em vista que o mesmo possa ter sido ingerido enquanto o item alimentar mais usual estava ausente, pouco freqüente ou difícil de ser capturado (Cyrus 1988, Pinnegar 2003). Para obter informações confiáveis acerca da escolha alimentar de peixes, é necessário analisar os conteúdos estomacais, a oferta de alimento e avaliar o comportamento alimentar através de observações diretas no ambiente natural e, se possível, também em condições experimentais (Zavala-Camin 1996).

Malacoctenus delalandii (Valenciennes, 1836) é uma espécie de pequeno porte, atingindo aproximadamente $8 \mathrm{~cm}$ de comprimento total, possui o focinho afilado, boca com dimensão moderada, o terceiro e o quarto espinhos da nadadeira dorsal menores que os demais (Carvalho-Filho 1999, Hostim-Silva et al. 2006). Habita os interstícios da porção rasa de costões rochosos, recifes de corais, bancos de algas e de areia, ocorrendo no Oceano Atlântico Ocidental, de Belize até Santa Catarina, e utiliza pequenos invertebrados em sua dieta, principalmente crustáceos (Hostim-Silva et al. 2006). Há alguns trabalhos que descrevem a composição da dieta de Malacoctenus, indicando um predomínio de crustáceos, particularmente decápodes (anomuros e braquiúros), anfípodes e isópodes, embora poliquetos e gastrópodes também sejam itens alimentares comumente utilizados (Cervigón 1966, Almeida 1972, Kotrschal \& Thomson 1986, Zamprogno 1989, Sabino 1992, Santos 2005).

O presente estudo teve como objetivo caracterizar a composição da dieta de $M$. delalandii na praia da Fortaleza, litoral norte do estado de São Paulo, comparar o conteúdo estomacal com a disponibilidade de itens alimentares encontrados no ambiente onde os indivíduos foram coletados, além de observar e descrever o comportamento alimentar da espécie.

\section{Material e Métodos}

\section{1. Área de estudo}

A região de Ubatuba constitui uma importante zona de transição faunística entre a Patagônia e regiões tropicais (Mantelatto 1995), com conjuntos de organismos adaptados às condições flutuantes do meio, havendo proporção significativa de elementos endêmicos, alguns membros das faunas adjacentes (Caribe e Patagônia) e organismos circuntropicais (Medeiros 1989). O estudo foi realizado na Praia da Fortaleza ( $23^{\circ} 32^{\prime} \mathrm{S}$ e $\left.45^{\circ} 10^{\prime} \mathrm{W}\right)$, no município de Ubatuba, estado de São Paulo (Figura 1). A praia localiza-se na enseada da Fortaleza formada por uma feição rochosa estreita que avança na direção sudoeste-nordeste, e apresenta uma grande variedade de substratos, representados principalmente por bancos de areia e rochas recobertas por algas calcárias da espécie Amphiroa beauvoisii (J.V. Lamouroux, 1816), algas frondosas como Sargassum filipendula e Galaxaura stupocaulon e pelo zoantídeo Palythoa caribaeorum (observação pessoal).

\section{Coleta de indivíduos e análise do conteúdo estomacal}

Em outubro de 2006 e março de 2007 indivíduos de Malacoctenus delalandii foram coletados com o uso de um puçá, através de técnica de mergulho livre ("snorkeling"). Os peixes foram capturados em águas rasas entre 0 e 2 metros de profundidade, nos períodos da manhã e da tarde, durante as quais foi registrada a atividade predatória da espécie (Zamprogno 1989).

Os indivíduos coletados foram anestesiados com mentol a 10 g.L. $\mathrm{L}^{-1}$ diluído na própria água do mar e fixados logo após a captura com solução de formaldeído a 10\%, injetada em suas cavidades abdominais para garantir a conservação do conteúdo estomacal (Zavala-Camin 1996). Em laboratório, os animais foram pesados e medidos (CT), os tubos digestivos foram retirados, colocados em placas de Petri e analisados em estereomicroscópio com aumento máximo de 40x. Os itens alimentares foram identificados até o menor nível taxo-

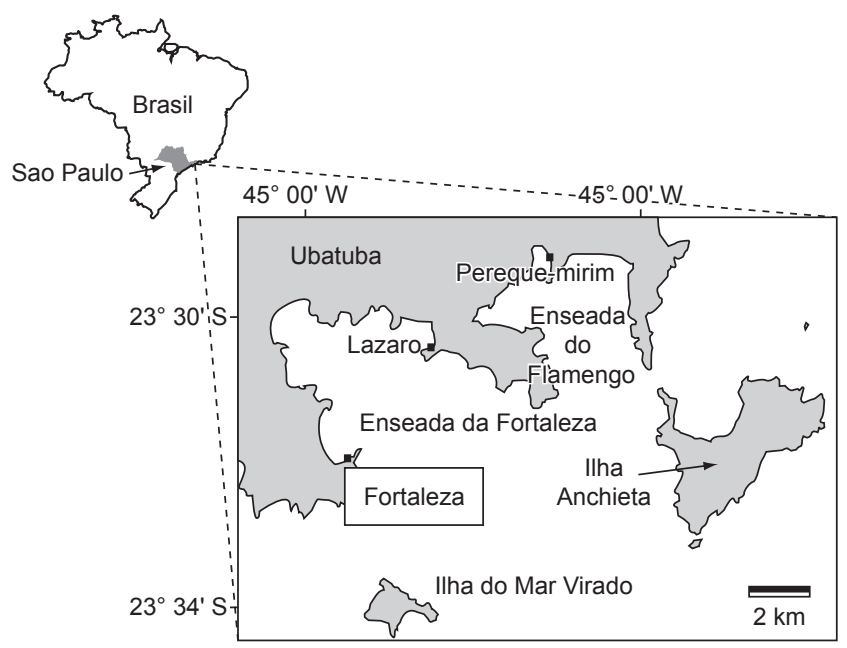

Figura 1. Localização da área de estudo, na Praia da Fortaleza $\left(23^{\circ} 32^{\prime} \mathrm{S}\right.$ e $45^{\circ} 10^{\prime}$ W), Ubatuba, Estado de São Paulo, Brasil.

Figure 1. Location of the study area, in Fortaleza Beach (23 $32^{\prime} \mathrm{S}$ and $\left.45^{\circ} 10^{\prime} \mathrm{W}\right)$, Ubatuba, State of São Paulo, Brazil. 
nômico possível, utilizando-se chaves específicas e consultando-se especialistas.

Para a análise qualitativa e quantitativa dos itens alimentares foi usado o método de frequiência de ocorrência (FO\%) (Hyslop 1980), segundo o qual, o número de peixes nos quais apareceu um determinado item é expresso como a porcentagem do número total de peixes examinados que contenham alimento. Como este método indica a constância ou a casualidade de cada tipo de item na dieta da espécie, foram estabelecidas três categorias para os itens alimentares (Zamprogno 1989) a) Itens "constantes", quando presentes em mais de 50\% dos estômagos; b) Itens "acessórios", quando presentes em 25 a $50 \%$ dos estômagos; and c) Itens "acidentais", quando presentes em menos de $25 \%$ dos estômagos. Calculou-se também a composição percentual $(\mathrm{CPE} \%)$, que equivale à porcentagem que cada item representa no total de itens encontrados nos estômagos (Hyslop 1980).

Além disso, foi utilizado o método da dominância (Hynes 1950, Bennemann et al. 2006), analisando-se a porcentagem do número de vezes que determinado item é dominante numericamente (ocorre mais vezes) em um estômago, em função do total de estômagos analisados.

\section{Disponibilidade de itens alimentares associados à alga Amphiroa beauvoisii}

A disponibilidade de itens alimentares associados à alga rodófita calcária Amphiroa beauvoisii, substrato predominante na área de estudo (dados não publicados) e utilizado por $M$. delalandii em sua atividade alimentar, foi comparada à composição dos conteúdos estomacais dos indivíduos amostrados. Para tanto, amostras aleatórias da alga foram coletadas em março de 2007, sendo cada amostra representada pelo material removido em um quadrante de $10 \times 10 \mathrm{~cm}$. As algas foram colocadas em sacos de tecido (voal) com malha de $0,25 \mathrm{~mm}$ e levadas ao laboratório. A fauna associada às algas foi removida e as algas secas à temperatura ambiente por 24 horas e, em seguida, pesadas. Posteriormente foram identificadas e quantificadas as espécies de invertebrados associadas e calculada a densidade (número de indivíduos por grama de alga). A composição percentual das espécies identificadas na alga e no conteúdo estomacal foi comparada através do teste G (Ayres et al. 2003).

\section{Observação do comportamento alimentar}

Para o estudo do comportamento alimentar também foi utilizada a técnica de mergulho livre. As observações foram realizadas do início da manhã (8:00 horas) até o final da tarde (17:00 horas). Nenhuma observação noturna foi realizada, pois não corresponde ao período de alimentação da espécie. As sessões de observação foram padronizadas em 10 minutos, exceto no caso de fuga dos indivíduos. Neste caso, a observação era interrompida e contabilizado o tempo até o momento da fuga. Inicialmente, foi utilizado para observação o método ad libitum (Altmann 1974, Lehner 1979), através do qual foi criada uma lista de padrões de comportamento alimentar apresentados pelos indivíduos de Malacoctenus delalandii. Posteriormente, foi utilizado o método animal focal, registrando-se todas as ocorrências do comportamento alimentar em uma prancheta de PVC. O comportamento dos indivíduos foi descrito e os seguintes dados foram registrados durante sessões de 10 (dez) minutos de observações subaquáticas para cada individuo: hora do início da observação, tipo de substrato no qual se iniciou o forrageamento e número de mordiscadas em cada tipo de substrato utilizado na alimentação (Ferreira et al. 1998, Bonaldo et al. 2005). Para comparar o número de mordiscadas por minuto (taxa de forrageamento) nos diferentes períodos do dia foi utilizado o teste de Kruskal-Wallis (Ayres et al. 2003).

\section{Resultados}

\section{Análise do conteúdo estomacal}

No total foram coletados 31 exemplares ( 15 em outubro de 2006 e 16 em março de 2007) com tamanho variando de 3,5 a 7,4 cm (CT), com média de $6,03 \mathrm{~cm}$ e peso total de 0,4 a 4,5 g. Dos 31 estômagos analisados, $29(93,5 \%)$ continham alimento e dois $(6,5 \%)$ estavam vazios.

Foram encontrados itens alimentares pertencentes a quatro filos, seis classes, seis ordens, 12 famílias, dois gêneros e nove espécies (Tabela 1). Segundo a classificação por categoria, foram considerados itens constantes aqueles pertencentes à Ordem Isopoda e à Subordem Gammaridea, ambos pertencentes à Superordem Peracarida. Os itens considerados acessórios foram a Classe Foraminiferida, a Classe Gastropoda, a Classe Ostracoda, o isópode Janaira gracilis, a Ordem Tanaidacea, a Família Ampithoidae, o gamarídeo Hyale nigra, e a Subordem Caprellidea - Caprella scaura. Os demais itens foram considerados acidentais.

Pôde-se verificar que no conteúdo estomacal da espécie foram encontrados invertebrados com dimensões e hábitos muito varia-

Tabela 1. Itens alimentares encontrados no conteúdo estomacal de Malacoctenus delalandii na praia da Fortaleza $(\mathrm{n}=29)$. $(\mathrm{FO} \%)=$ freqüência de ocorrência. (d) = número de estômagos em que determinado item alimentar foi dominante. $(\mathrm{D} \%)=$ porcentagem de dominância. Categorias $=$ categoria da constância de ocorrência. ( ) = número de estômagos em que o item foi encontrado.

Table 1. Food items found in the stomach contents of Malacoctenus delalandii on the Fortaleza beach $(\mathrm{n}=29)$. $(\mathrm{FO} \%)=$ frequency of occurrence. $(\mathrm{d})$ $=$ number of stomachs in which a certain item was dominant. $(\mathrm{D} \%)=$ percentage of dominance. Categories $=$ categories of occurrence constancy. $($ ) $=$ number of stomachs in which the item was found.

\begin{tabular}{|c|c|c|c|c|}
\hline Item alimentar & F.O. $(\%)$ & d & D (\%) & Categorias \\
\hline FILO Protozoa - Foraminiferida - Foraminífero (9) & 31,0 & - & - & Acessório \\
\hline FILO Mollusca - Classe Gastropoda (8) & 27,6 & - & - & Acessório \\
\hline Fragmento Gastropoda (3) & 10,4 & - & - & - \\
\hline Ordem Archaeogastropoda & - & - & - & - \\
\hline Fissurella sp. (1) & 3,4 & - & - & - \\
\hline Ordem Mesogastropoda & - & - & - & - \\
\hline Familia CAECIDAE (4) & - & - & - & - \\
\hline Caecum sp. (4) & 13,8 & - & - & - \\
\hline FILO Annelida - Classe Polychaeta (7) & 24,1 & 2 & 6,9 & Acidental \\
\hline FILO Arthropoda - Classe Insecta (1) & 3,4 & - & - & Acidental \\
\hline
\end{tabular}


Tabela 1. Continuação...

\begin{tabular}{|c|c|c|c|c|}
\hline Item alimentar & F.O. $(\%)$ & d & $\mathrm{D}(\%)$ & Categorias \\
\hline FILO Arthropoda - Crustacea & 38,0 & 5 & 17,2 & Acessório \\
\hline Classe Ostracoda (11) & - & - & - & - \\
\hline FILO Arthropoda - Crustacea & 17,2 & - & - & Acidental \\
\hline Classe Copepoda (5) & - & - & - & - \\
\hline FILO Arthropoda - Crustacea & - & - & - & - \\
\hline Classe Malacostraca & - & - & - & - \\
\hline Superordem Peracarida & - & - & - & - \\
\hline Ordem Decapoda (2) & 6,9 & - & - & Acidental \\
\hline FILO Arthropoda - Crustacea & - & - & - & - \\
\hline Classe Malacostraca & 75,8 & - & - & - \\
\hline Superordem Peracarida & 55,2 & - & - & - \\
\hline Ordem Isopoda (22) & - & 8 & 27,6 & Constante \\
\hline Fragmento Ordem Isopoda (16) & 38,0 & 5 & 17,2 & - \\
\hline Família JANIRIDAE (11) & - & - & - & - \\
\hline Janaira gracilis (11) & - & 2 & 6,9 & - \\
\hline Família JAEROPSIDAE (1) & - & - & - & - \\
\hline Jaeropsis dubia (1) & 3,4 & - & - & - \\
\hline Família SHAEROMATIDAE (5) & - & - & - & - \\
\hline Cymodoce brasiliensis (5) & 17,2 & 1 & 3,4 & - \\
\hline FILO Arthropoda - Crustacea & - & - & - & - \\
\hline Classe Malacostraca & - & - & - & - \\
\hline Superordem Peracarida & - & - & - & - \\
\hline Ordem Tanaidacea (12) & 41,4 & 2 & 6,9 & Acessório \\
\hline Fragmento Ordem Tanaidacea (11) & 38,0 & 2 & 6,9 & - \\
\hline Familia LEPTOCHELIIDAE (1) & - & - & - & - \\
\hline Leptochelia savignyi (1) & 3,4 & - & - & - \\
\hline FILO Arthropoda - Crustacea & - & - & - & - \\
\hline Classe Malacostraca & - & - & - & - \\
\hline Superordem Peracarida & - & - & - & - \\
\hline Ordem Amphipoda & - & - & - & - \\
\hline Subordem Gammaridea (25) & 86,2 & 11 & 37,8 & Constante \\
\hline Fragmento Subordem Gammaridea (17) & 58,6 & 6 & 20,7 & - \\
\hline Família AMPHILOCHIDAE (3) & - & - & - & - \\
\hline Amphilocus neapolitanus (3) & 10,4 & - & - & - \\
\hline Família AMPITHOIDAE (14) & 48,3 & 3 & 10,4 & - \\
\hline Cymadusa filosa (2) & 6,9 & - & - & - \\
\hline Família HYALIDAE (9) & - & - & - & - \\
\hline Hyale nigra $(9)$ & 31,0 & 2 & 6,9 & - \\
\hline Família MELITIDAE (1) & 3,4 & - & - & - \\
\hline Família STENOTHOIDAE (1) & - & - & - & - \\
\hline Stenothoe valida (1) & 3,4 & - & - & - \\
\hline FILO Artropoda - Crustacea & - & - & - & - \\
\hline Classe Malacostraca & - & - & - & - \\
\hline Superordem Peracarida & - & - & - & - \\
\hline Ordem Amphipoda & - & - & - & - \\
\hline Subordem Caprellidea (12) & 41,4 & 1 & - & Acessório \\
\hline Fragmento Subordem Caprellidea (10) & 34,5 & - & 3,4 & - \\
\hline Família Caprellidae (12) & - & - & - & - \\
\hline Caprella scaura (12) & 41,4 & 1 & 3,4 & - \\
\hline Fragmentos de Alga Verde (9) & 31,0 & - & - & Acessório \\
\hline Fragmentos de Alga Calcárea (9) & 31,0 & - & - & Acessório \\
\hline
\end{tabular}


dos, desde foraminíferos com tamanho extremamente reduzido até caprelídeos com dimensões bem maiores.

Adicionalmente, foi utilizado o método da dominância, que é uma alternativa à pesagem dos itens, devido às dimensões muito pequenas e o avançado estado de digestão dos mesmos. Observando os valores de dominância pôde-se notar que alguns itens com valores não muito altos de freqüência de ocorrência e de composição percentual foram dominantes em vários estômagos analisados. É o caso do item Ostracoda, que teve uma frequiência de ocorrência de 38,0\% e uma composição percentual baixa de $8,0 \%$ e, no entanto, foi dominante em cinco $(17,2 \%)$ dos estômagos analisados.

Através da representação gráfica da composição percentual (CPE\%) (Figura 2), que corresponde a quanto cada item representa no total de itens encontrados, na dieta de Malacoctenus delalandii, pôde-se perceber que os itens Gammaridea e Isopoda foram os mais importantes, representando 18,0 e 17,0\% respectivamente. Já os itens Insecta e Decapoda tiveram representações mínimas, com 1,0 e 2,0\% respectivamente. Demais itens, como Tanaidacea e Caprellidea que apresentaram valores relevantes na freqüência de ocorrência, também podem ser considerados importantes na composição percentual, pois representaram cada um $9,0 \%$ dos itens encontrados.

\section{Disponibilidade de itens alimentares associados à alga Amphiroa beauvoisii}

A disponibilidade de itens alimentares associados à alga rodófita calcária Amphiroa beauvoisii, a qual Malacoctenus delalandii está intimamente relacionado, foi avaliada comparando-se os itens encontrados nos estômagos dos indivíduos coletados com as amostras coletadas da alga. Foram coletadas dez amostras de algas, que totalizaram 77,25 g, nas quais foram encontrados 4.668 invertebrados o que resultou em uma densidade média de 60,42 indivíduos. $\mathrm{g}^{-1}$.

Houve uma grande variação na densidade dos invertebrados encontrados nas algas (Tabela 2). Alguns apresentaram densidades mínimas, como é o caso da Classe Echinoidea $\left(0,018 \pm 0,056\right.$ ind. $\left.\mathrm{g}^{-1}\right)$

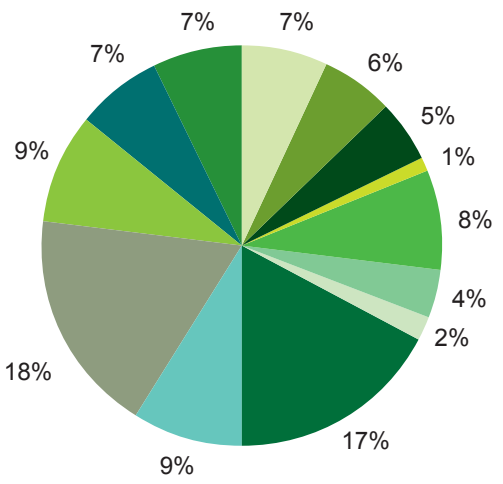

\begin{tabular}{|lll|}
\hline Foraminiferida & $\square$ Polychaeta & Ostracoda \\
Decapoda & $\square$ Tanaidacea & Caprellidea \\
$\square$ Frag. Alga Calcárea & $\square$ Gastropoda & $\square$ Insecta \\
Copepoda & $\square$ Isopoda & Gammaridea \\
Frag. Alga verde & & \\
\hline
\end{tabular}

Figura 2. Composição percentual (CPE\%) dos conteúdos estomacais de Malacoctenus delalandii com os itens alimentares agrupados em categorias amplas.

Figure 2. Percent composition (CPE\%) of the stomach contents of the Malacoctenus delalandii with the food items grouped in broad categories. e Holothurioidea $\left(0,09 \pm 0,029\right.$ ind. $\left.\mathrm{g}^{-1}\right)$ e também da Ordem Decapoda $\left(0,010 \pm 0,034\right.$ ind. $\left.\mathrm{g}^{-1}\right)$, no entanto outros táxons foram encontrados em grande quantidade em todas as algas, como é o caso do gastrópode Caecum sp. com densidade de 13,10 $\pm 15,42$ ind. ${ }^{-1}$ e da Classe Polychaeta $\left(11,18 \pm 5,898\right.$ ind. $\left.\mathrm{g}^{-1}\right)$.

Ao avaliar a composição percentual dos invertebrados encontrados nas algas calcárias e nos conteúdos estomacais dos indivíduos coletados em março de 2007, pôde-se concluir que houve diferença significativa em sua ocorrência. Tal diferença foi observada para grandes grupos $(\mathrm{G}=278,89$; g.L. $=70 ; \mathrm{p}<0,01)$, com maior incidência de itens dos grupos Gammaridea, Caprellidea, Isopoda e Ostracoda. Por outro lado, grupos como o filo Echinodermata (Classes Ophiuroidea, Echinoidea e Holothurioidea), a classe Pycnogonida e a família Chironomidae (Diptera) foram encontrados exclusivamente na alga calcária (Figura 3a).

No entanto, ao avaliar somente as espécies de crustáceos peracáridos, não foi verificada diferença significativa $(\mathrm{G}=87,31$; g.L. $=44$; $\mathrm{p}>0,05)$ entre a composição percentual $(\mathrm{CPE} \%)$ dos invertebrados encontrados nas algas calcárias e nos conteúdos estomacais de Malacoctenus delalandii (Figura 3b).

\section{Comportamento alimentar}

O comportamento alimentar da espécie foi observado em 44 indivíduos, com sessões de 10 minutos (excetuando-se fuga), obtendo-se um total de 410 minutos de observação direta (6,8 horas).

Pôde-se verificar que a espécie utiliza a tática de especulação do substrato "grubber excavation while moving" como tipo de estratégia alimentar. Os indivíduos geralmente permanecem abrigados em tocas ou fendas próximas às regiões com grande disponibilidade de alga calcária e se deslocam próximos ao substrato, capturando através de movimentos curtos e rápidos invertebrados associados às algas. Verificou-se durante as observações que os indivíduos não percorrem grandes distâncias para explorar os substratos de alga calcária e capturar invertebrados. A distância média percorrida foi de $82,19 \mathrm{~cm}$, com valor mínimo de $30 \mathrm{~cm}$ e máximo de $200 \mathrm{~cm}$.

Foi observado em 31 indivíduos $(70,45 \%)$ um comportamento particular de expulsão de partículas ao mordiscar o substrato e capturar o alimento.

Verificou-se também uma variação na coloração dos indivíduos, dependendo da coloração dos substratos nos quais os mesmos se encontravam, variando de vermelho escuro (locais onde as algas são mais escuras) a um marrom claro em locais com menos algas e mais rochas. O substrato dominante no início do forrageamento foi a alga calcária $(95,4 \%)$, no entanto, o peixe também foi encontrado sob rochas $(2,3 \%)$ e no fundo arenoso $(2,3 \%)$.

A taxa média de forrageamento (número de mordiscadas por minuto) no substrato de alga calcária (Figura 4) foi de 0,74 $\pm 0,164$ mordicadas por minuto (média \pm desvio padrão). Não houve diferença significativa na taxa de forrageamento de Malacoctenus delalandii entre os diferentes períodos do dia $(H=9,740$; g.L. $=8 ; \mathrm{p}>0,05)$.

Foi observada em duas oportunidades durante os mergulhos, a tentativa de caranguejos (Brachyura) adultos capturarem os indivíduos de Malacoctenus delalandii, enquanto os mesmos exploravam o substrato de alga calcária e tentavam encontrar os invertebrados para alimentação, principalmente em locais com frestas e tocas.

\section{Discussão}

O hábito alimentar carnívoro é bastante difundido entre os representantes da família Labrisomidae, como atestam diversos trabalhos realizados em diferentes regiões. Randall (1967) encontrou principalmente decápodes e peracáridos na dieta de Labrisomus nuchipinnis e Labrisomus guppyi na América Central. O trabalho de Zamprogno 
Tabela 2. Lista de espécies e densidade dos invertebrados encontrados nas amostras de Amphiroa beauvoisii coletadas na praia da Fortaleza, município de Ubatuba. Densidade (média \pm desvio padrão) $=$ Ind. $\mathrm{g}^{-1}$

Table 2. List of species and density of the invertebrates found in samples of Amphiroa beauvoisii collected on Fortaleza beach, Ubatuba. Density (average \pm standard deviation $)=$ Ind. $\mathrm{g}^{-1}$

\begin{tabular}{|c|c|c|c|c|c|}
\hline Filo & Classe & Ordem & Família & Espécie & Densidade \\
\hline Protozoa & - & Foraminiferida & - & - & $2,761 \pm 2,523$ \\
\hline \multirow[t]{10}{*}{ Mollusca } & Gastropoda & Archeogastropoda & FISSURELIDAE & Fissurella sp. & $0,472 \pm 0,529$ \\
\hline & & - & SCISSURELLIDAE & Scissurella cingulata & $1,157 \pm 2,218$ \\
\hline & & Mesograstropoda & TRICOLIIDAE & Tricolia affinis & $9,839 \pm 11,66$ \\
\hline & & - & CAECIDAE & Caecum sp. & $13,10 \pm 15,42$ \\
\hline & & - & CERITHIIDAE & Cerithium atratum & $0,817 \pm 0,661$ \\
\hline & & Neogastropoda & NATICIDAE & Natica sp. & $0,057 \pm 0,103$ \\
\hline & & & COLUMBELLIDAE & Anachis sp. & $0,158 \pm 0,156$ \\
\hline & & Nudibranchia & PYRAMIDELLIDAE & Odostomia impressa & $0,187 \pm 0,190$ \\
\hline & & - & - & - & $0,205 \pm 0,285$ \\
\hline & Bivalvia & - & - & - & $0,384 \pm 0,374$ \\
\hline Annelida & Polychaeta & - & - & - & $11,18 \pm 5,898$ \\
\hline \multirow[t]{19}{*}{ Artropoda } & Malacostraca & Amphipoda & AMPITHOIDAE & Ampithoe ramondi & $0,090 \pm 0,020$ \\
\hline & & - & - & Cymadusa filosa & $0,125 \pm 0,228$ \\
\hline & & - & HYALIDAE & Hyale nigra & $0,780 \pm 1,421$ \\
\hline & & - & LYSIANASSIDAE & Shoemakerella nasuta & $0,093 \pm 0,196$ \\
\hline & & - & MELITIDAE & Dulichiella appendiculata & $0,210 \pm 0,318$ \\
\hline & & - & - & Elasmopus brasiliensis & $1,847 \pm 1,604$ \\
\hline & & - & - & Quadrimaera miranda & $0,015 \pm 0,047$ \\
\hline & & - & CAPRELLIDAE & Caprella scaura & $0,068 \pm 0,123$ \\
\hline & & Decapoda & XANTHIDAE & Eurypanopeus abbreviatus & $0,010 \pm 0,034$ \\
\hline & & - & HIPPOLYTIDAE & Hippolyte sp. & $0,114 \pm 0,262$ \\
\hline & & Isopoda & ANTHURIDAE & Paranthura urochroma & $0,140 \pm 0,186$ \\
\hline & & - & JANIRIDAE & Janaira gracilis & $1,604 \pm 1,198$ \\
\hline & & - & SPHAEROMATIDAE & Cymodoce brasiliensis & $0,033 \pm 0,104$ \\
\hline & & Tanaidacea & LEPTOCHELIIDAE & Leptochelia savignyi & $4,764 \pm 5,431$ \\
\hline & & & TANAIDAE & Zeuxo coralensis & $0,043 \pm 0,135$ \\
\hline & Ostracoda & - & - & - & $5,567 \pm 10,12$ \\
\hline & Maxillopoda & Harpacticoida & PELTIDIIDAE & - & $0,009 \pm 0,029$ \\
\hline & Pycnogonida & - & - & - & $0,034 \pm 0,058$ \\
\hline & Insecta & Diptera & CHIRONOMIDAE & - & $0,633 \pm 1,083$ \\
\hline \multirow[t]{4}{*}{ Echinodermata } & Ophiuroidea & Ophiurida & AMPHIURIDAE & Amphipholis squamata & $4,204 \pm 3,429$ \\
\hline & & & OPHIACTIDAE & Ophiactis savignyi & $1,486 \pm 1,378$ \\
\hline & Echinoidea & Temnopleuroida & TOXOPNEUSTIDAE & Lytechinus variegatus & $0,018 \pm 0,056$ \\
\hline & Holothurioidea & - & - & - & $0,009 \pm 0,029$ \\
\hline
\end{tabular}
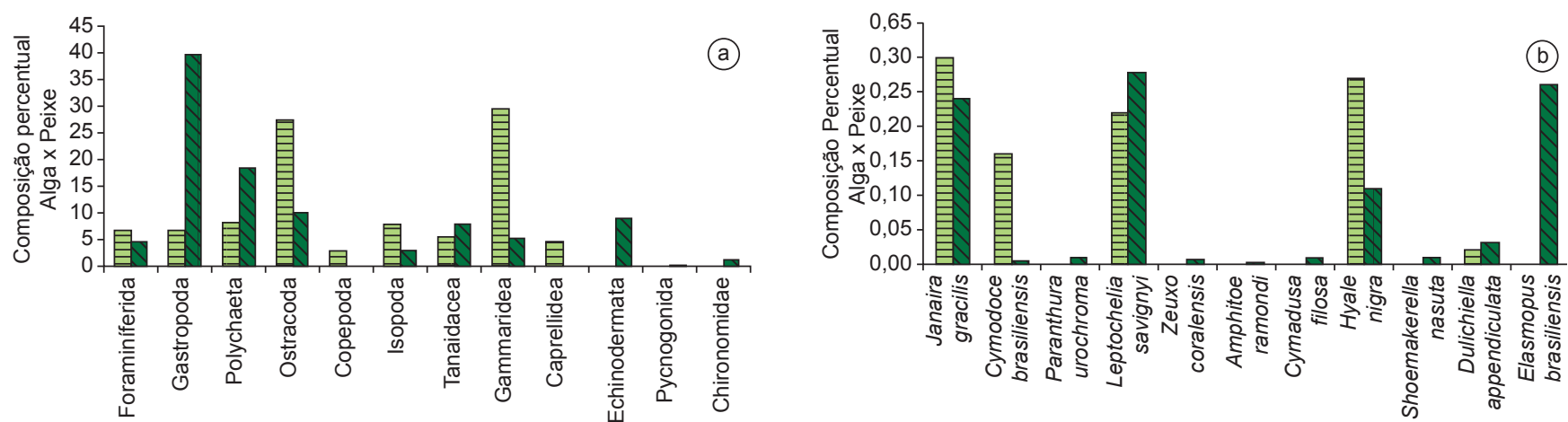

$\exists$ Peixe $\quad \square$ Alga

Figura 3. a) Composição percentual (CPE\%) dos invertebrados agrupados em categorias amplas e dos b) crustáceos peracáridos encontrados nas algas calcárias e conteúdos estomacais de Malacoctenus delalandii, durante a coleta realizada em março 2007.

Figure 3. a) Percent composition (CPE\%) of the invertebrates grouped in broad categories and of b) peracarid crustaceans found in the calcareous algae and stomach contents of the Malacoctenus delalandii, in March 2007 sampling. 


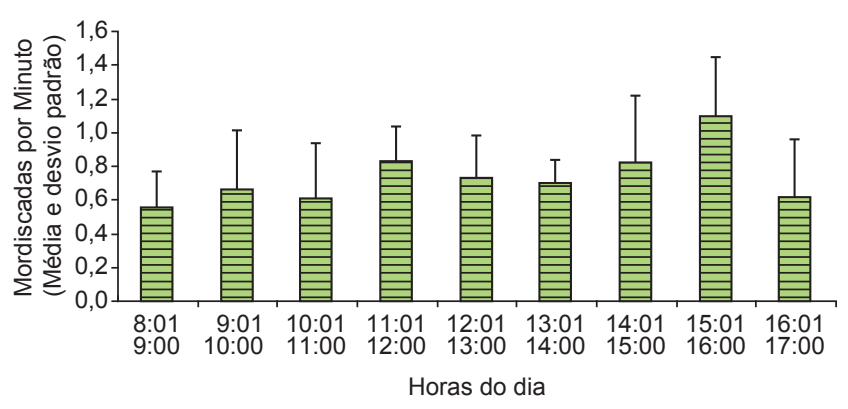

Figura 4. Taxa de forrageamento (mordiscadas por minutos) no substrato de alga calcária, para indivíduos de Malacoctenus delalandii $(\mathrm{N}=44)$.

Figure 4. Foraging rate (bites per minutes) on the calcareous algal substrate, for Malacoctenus delalandii individuals $(\mathrm{N}=44)$.

(1989), no litoral do Espírito Santo, descreveu principalmente poliquetas, isópodes e anfípodes nos tubos digestivos de Labrisomus nuchipinnis. Quijada \& Cáceres (2000), na costa do Chile, encontraram crustáceos decápodes como principal item na alimentação dos labrisomídeos Auchenionchus variolosus e Calliclinus geniguttatus. Também para uma espécie da família Labrisomidae, endêmica das ilhas Galápagos, Dialommus fuscus, Nieder (2001) confirmou o hábito carnívoro e encontrou principalmente decápodes e copépodes, além de anfípodes, isópodes e poliquetas no conteúdo estomacal. Kotrschal \& Thomson (1986), analisando o conteúdo estomacal de cinco espécies do gênero Malacoctenus encontrou anfípodes, caranguejos anomura e braquiúra, poliquetas errantes e Caprellidea.

Baseando-se nas análises de conteúdo estomacal, Malacoctenus delalandii também pode ser considerada uma espécie de hábito alimentar carnívoro, alimentando-se de uma grande variedade de invertebrados, principalmente itens pertencentes à Superordem Peracarida, observando-se uma freqüência de ocorrência de 75,8\% para isópodes e de $86,2 \%$ para anfípodes. As informações disponíveis na literatura sobre hábitos alimentares de Malacoctenus delalandii também indicam o hábito carnívoro e corroboram os dados obtidos no presente estudo. Em estudo realizado por Cervigón (1966) na costa da Venezuela foram encontrados principalmente pequenos crustáceos decápodes e anfípodes nos conteúdos estomacais de M. delalandii. Já no Brasil, Almeida (1972), na região de Salvador, Bahia, encontrou fragmentos de crustáceos na dieta de $M$. delalandii. Também no Brasil, no litoral do Espírito Santo, Zamprogno (1989) encontrou anfípodes e isópodes como itens principais. Sabino (1992), estudando a partilha de recurso alimentar e espacial em duas espécies de labrisomídeos, em costões rochosos do litoral de Ubatuba, SP, descreveu que a dieta desta espécie é composta basicamente de Crustacea (Amphipoda \& Majidae), Polychaeta e Gastropoda. Santos (2005), em estudo da ecologia trófica de espécies de Blennioidei em São Sebastião, SP, descreveu que a dieta de $M$. delalandii é composta principalmente por anfípodes e larvas de crustáceos.

Os táxons mais importantes encontrados em algas calcárias e consumidos por comunidades de peixes bentívoros carnívoros são Crustacea e Polychaeta (Norton \& Benson 1983, De Grave et al. 2000, Foster 2001), fato confirmado no presente estudo. Os gamarídeos tiveram a maior ocorrência no conteúdo estomacal, o que ocorre, provavelmente, devido à grande representatividade desses crustáceos em comunidades associadas a algas bentônicas (Nakamura 1971, Tararam \& Wakabara 1981, Jacobucci \& Leite 2002), incluindo-se substratos dominados por algas calcárias (Masunari 1983, Metri 2006). O item Isopoda foi o segundo em frequiência de ocorrência nos conteúdos estomacais, o que também é justificado por serem crustáceos predominantemente bentônicos e de ampla ocorrência nos substratos. Além disso, os isópodes representam também um dos principais itens encontrados no conteúdo estomacal de peixes recifais de regiões temperadas (Nakamura 1971).

Embora tenha sido encontrada uma freqüência de ocorrência nos estômagos de $31 \%$ de algas calcárias e também de $31 \%$ de algas verdes, tal fato não as caracteriza como item acessório, pois são ingeridas acidentalmente no momento que a espécie captura os invertebrados que forrageiam sobre as algas e assim, não fazem parte diretamente do hábito alimentar do peixe (Linkowski et al. 1983, Kotrschal \& Thomson 1986, Santos 2005).

Foi observada nas análises do conteúdo estomacal, para alguns itens, uma dominância elevada associada a uma freqüência de ocorrência baixa. Isso demonstra que determinado item não esteve presente em um grande número de estômagos, no entanto, quando presente ocorreu em grandes quantidades. Isto pode indicar que o item é raro ou tem distribuição heterogênea no substrato, fato comum em comunidades bentônicas (Tanaka et al. 2003) e, no entanto, faz parte da dieta da espécie. Tal situação fica evidente com o item Ostracoda, que atingiu valores altos de dominância comparada à frequiência de ocorrência.

Peixes que vivem associados a ambientes recifais apresentam um significativo padrão de seletividade alimentar, que ainda não foi claramente compreendido, no entanto, seguem alguns princípios relacionados à qualidade nutricional, anatomia e comportamento da presa, além da morfologia no predador (Keesing 1990, Gochfeld 2004, Pratchett 2007). As análises obtidas, comparando os invertebrados encontrados nos estômagos dos peixes com os do substrato de alga calcária, demonstraram que existe diferença significativa na composição da dieta desta espécie em relação à oferta alimentar encontrada na alga rodófita calcária Amphiroa beauvoisii. Alguns itens como o gastrópode Tricolia affinis, e os ofiuróides Amphipholis squamata e Ophiactis savignyi possuíram altos valores de densidade (ind. $\mathrm{g}^{-1}$ ) no substrato, no entanto, não foram encontrados em nenhum estômago. A não utilização de Amphipholis squamata e Ophiactis savignyi pode estar relacionada à impalatabilidade desses itens e principalmente ao tamanho relativamente grande em relação à abertura da boca de Malacoctenus delalandii (Main et al. 1985, Pinnegar et al. 2003). Já no caso do gastrópode Tricolia affinis, o fato pode estar relacionado à baixa qualidade nutricional do alimento, devido à presença da concha calcária que não teria um importante papel na dieta da espécie (Pratchett 2007).

Outros itens não apresentaram valores de densidade tão elevados na alga calcária Amphiroa beauvoisii, no entanto, foram muito abundantes nos conteúdos estomacais, podendo-se citar como exemplo, os itens Caprella scaura (anfípode) e Janaira gracilis (isópode). Isto indica que o consumo significativo desses itens pela espécie pode estar relacionado à alta qualidade nutricional dos mesmos (Gochfeld, 2004) ou à facilidade de captura das presas. Anfípodes caprelídeos, embora possam apresentar características crípticas em relação ao substrato em que se fixam, têm baixa mobilidade (Caine 1989), e são relativamente grandes, quando comparados a outros invertebrados associados a algas, o que poderia explicar a preferência de $M$. delalandii por esses peracáridos.

Em ambientes recifais tropicais, as espécies podem ser ativas durante o dia (diurnas), durante a noite (noturnas) ou ainda durante o crepúsculo (crepusculares) (Collette \& Talbot 1972, ligo \& Tabata 1996). Segundo Hobson (1973), quando estes peixes estão ativos suas ações são dominadas pela alimentação, e quando estão inativos, seu comportamento está relacionado preferencialmente com a proteção. Malacoctenus delalandii pode ser caracterizada como uma espécie bentônica recifal de hábito diurno, o que é confirmado por estudos 
anteriores no Brasil (Menezes \& Figueiredo 1985, Zamprogno 1989, Sabino 1992, Carvalho-Filho 1999, Santos 2005).

Com relação ao comportamento alimentar, concluiu-se através das observações subaquáticas que $M$. delalandii utiliza a estratégia de especulação do substrato ("grubber excavation while moving"). Essa estratégia alimentar já havia sido observada por Santos (2005), na região de São Sebastião, litoral norte do estado de São Paulo. Embora essa estratégia seja utilizada por outros representantes da família Labrisomidae, não é a única. Labrisomus nuchipinnis, por exemplo, é um típico predador de espera ("sit and wait predator") (Sazima 1986, Santos 2005).

O fato dos indivíduos observados não percorrerem grandes distâncias para explorarem os substratos de alga calcária (a distância média percorrida foi de $82,19 \mathrm{~cm}$ ) e utilizarem com frequiência frestas e tocas sugere que os indivíduos da espécie devem apresentar comportamento territorial e hábito criptobêntico, o que já foi relatado para várias espécies de peixes recifais bentônicos (Mendes 2006) e para outras espécies da família Labrisomidae (Nieder 2001). Sabino (1992) observou o comportamento territorial de Labrisomus nuchipinnis e Malacoctenus delalandii, inclusive medindo o território de ambas as espécies, obtendo valores baixos e relacionando a territorialidade com a proteção do recurso alimentar. $\mathrm{O}$ fato dos indivíduos percorrerem pequenas distâncias pode estar associado à territorialidade ou também à pressão de predação (Santos 2005), que pode ser exercida por outros peixes e por Brachyura, como observado no presente estudo.

Não há na literatura trabalhos que descrevam a taxa de forrageamento de espécies da família Labrisomidae. No entanto, comparandose com outras espécies recifais, Chaetodon striatus pertencente à família Chaetodontidae (Bonaldo et al. 2005) e Umbrina coroides à família Sciaenidae (Zahorcsak et al. 2000) notou-se uma atividade alimentar (número de mordiscadas por minuto) bem menor. Enquanto $M$. delalandii apresentou uma taxa de 0,74 $\pm 0,164$ mordicadas per minutes (média \pm desvio padrão), Chaetodon striatus apresentou um valor de 1,60 \pm 1,76 mordicadas per minutes (média \pm desvio padrão) e Umbrina coroides um valor de 7,9 $\pm 1,9$ mordicadas per minutes. Essa diferença tão evidente pode ser explicada pela pouca atividade da espécie e por serem preferencialmente criptobênticos.

Assim, concluiu-se que Malacoctenus delalandii é uma espécie de hábitos alimentares carnívoros, com dieta composta principalmente por crustáceos peracáridos e que utiliza estratégia alimentar de especulação do substrato "grubber excavation while moving". Trata-se de uma espécie com reduzida taxa de forrageamento e que não apresenta picos de atividade alimentar ao longo do dia.

\section{Agradecimentos}

Agradecemos Sérgio Magalhães Rezende pela leitura do manuscrito e relevantes sugestões, à Profa. Dra. Alessandra Pereira Majer pela identificação dos ofiuróides e aos biólogos Jairo Zancaner, Henrique Grande e Mauro Tavares pelo auxílio nas coletas. Aos revisores anônimos que também auxiliaram e engrandeceram o trabalho.

\section{Referências Bibliográficas}

ALMEIDA, V.G. 1972. Contribuição para o conhecimento dos peixes de poças de maré de Salvador e adjacências. Dissertação de Mestrado. Universidade de São Paulo, São Paulo, SP.

ALTMANN, J. 1974. Observational study of behaviour: sampling methods. Behaviour, 49:227-265.

AMARAL, A.C.Z. \& MIGOTTO, A. 1980. Importância dos anelídeos poliquetas na alimentação da macrofauna demersal e epibentônica da região de Ubatuba. Bol. Inst. Oceanogr. 29(2):31-35.
AYRES, M., AYRES, M.J.R., AYRES, D.L. \& SANTOS, A.A.S. 2003. BioEstat 3.0: aplicações estatísticas nas áreas de Ciências Biológicas e Médicas. Sociedade Civil Mamirauá, Belém.

BENNEMANN, S.T., CASATTI, L. \& OLIVEIRA, D.C. 2006. Alimentação de peixes: proposta para análise de itens registrados em conteúdos gástricos. Biota Neotrop. 6(2): http://www.biotaneotropica.org.br/v6n2/ pt/abstract?article+bn01206022006.

BONALDO, R.M., KRAJEWSKI, J.P. \& SAZIMA, I. 2005. Meals for two: foraging activity of the butterflyfish Chaetodon striatus (Perciformes) in southeast Rev. Bras. Biol. = Braz. J. Biol. 65(2):01-06.

CAINE, E.A. 1989. Caprellid amphipod behaviour and predatory strikes by fish. J. Exp. Mar. Biol. Ecol. 126(2):173-180.

CARVALHO-FILHO, A. 1999. Peixes: costa brasileira. 3 ed. Editora Melro, São Paulo.

CERVIGÓN, F. 1966. Los peces marinos de Venezuela. Vols I e II. Fundacion La Salle, Carac

COLLETTE, B.B. \& TALBOT, F.H. 1972. Activity patterns of coral reeffishes with emphasis on nocturnal-diurnal changeover. B. Am. Mus. Nat. Hist. 14:98-124.

COSTA, T.L.M., VASCONCELOS FILHO, A.L. \& VIANA, E.M.B.G. 1987. Aspectos gerais sobre a alimentação do Cangulu, Balistes ventula Linnaeus, 1758 (Pisces - Balistidae) no Estado de Pernambuco. Revta. bras. Zool. 4(2):71-88.

CYRUS, D.P. 1988. Episodic events and estuaries: of cyclonic flushing on the benthic fauna and diet of Solea bleekeri (Teleostei) in lake St. Lucia on the south-eastern coast of Africa. J. Fish Biol. 33(suppl. A):1-7.

DE GRAVE, S., FAZAKERLEY, H., KELLY, L., GUIRY, M.D., RYAN, M. \& WALSHE, J. 2000. A study of selected maërl beds in Irish waters and their potential for sustainable extraction. The Marine Institute, Dublin.

EDGAR, G.J. \& SHAW, C. 1995. Diets of fishes and trophic relationships between fishes and benthos at Western Port, Victoria. J. Exp. Mar. Biol. Ecol. 194(1):83-106.

FERREIRA, C.E.L., GONÇALVES, J.E.A., COUTINHO, R. \& PERET, A.C. 1998. Herbivory by the dusky damselfish Stegastes fuscus (Cuvier, 1830) in a tropical rocky shore: effects on the benthic community. J. Exp. Mar. Biol. Ecol. 229(2):241-264.

FOSTER, M. 2001. Rhodoliths: between rocks and soft places. J. Phycol. 37(5):659-667.

GERKING, S.D. 1994. Feeding ecology of fish. San Diego Academic Press, California.

GOCHFELD, D.J. 2004. Predation-induced morphological and behavioral defenses in a hard coral: implications for foraging behavior of coralfeeding butterflyfishes. Mar. Ecol. Progr. Ser. 267:145-158.

HAHN, N.S. \& DELARIVA, R.L. 2003. Métodos para avaliação da alimentação natural de peixes: o que estamos usando? Interciência 28(2): 100-104.

HOBSON, E.S. 1973. Diel feeding migrations in tropical reef fishes. Helgolander wiss. Meeresunters, 24(1):361-370.

HOBSON, E. S. \& CHESS, J. R. 1986. Relationships among fishes and their prey in a nearshore sand community of southern California. Environ. Biol. Fish. 17(3):201-226.

HOSTIM-SILVAM.,ANDRADE,A.B., MACHADO, L.F, GERHARDINGER, L.C., DAROS, F.A., BARREIROS, J.P. \& GODOY, E. 2006. Peixes de Costão Rochoso de Santa Catarina, I. Arvoredo. UNIVALI Edições, Itajaí, SC.

HYNES, H.B.N. 1950. The food of fresh-water sticklebacks (Gasterosteus aculeatus and Pygosteus pungitius), with a review of methods used in studies of the food of fishes. J. Anim. Ecol. 19:36-57.

HYSLOP, E.J. 1980. Stomach contents analysis - a review of methods and their application. J. Fish Biol. 17(4):411-429.

IIGO, M. \& TABATA, M. 1996. Circadian rhythms of locomotor activity in the goldfish Carassius auratus. Physiol. Behav. 60(3):775-781.

JACOBUCCI, G. B. \& LEITE, F. P. P. 2002. Distribuição vertical e flutuação sazonal da macrofauna vágil associada a Sargassum cymosum C. Agardh, 
na praia do Lázaro, Ubatuba, São Paulo, Brasil. Revta. bras. Zool., 19 (supl. 1), 87-100.

KAWAKAMI, E. \& AMARAL, A.C.Z. 1983. Importância dos anelídeos poliquetos no regime alimentar de Etropus longimanus (Norman, 1933) e Symphurus jenynsi (Evermann Kendall, 1907) (Pisces, Pleuronectiformes). Iheríngia. Ser. Zool. 62(1):47-54.

KEESING, J. K. 1990 Feeding biology of the crown-of-thorns starfish, Achanthaster planci (Linnaeus). Phd Thesis. James Cook University, Townsville.

KOTRSCHAL, K. \& THOMSON, D.A. 1986. Feeding patterns in eastern tropical Pacific blennioid fishes (Teleostei: Tripterygiidae, Labrisomidae, Chaenopsidae, Blenniidae). Oecologia, 70(3):367-378.

LEHNER, P. 1979. Handbook of ethological methods. S.T.P.M. Press, Garland, New York.

LINKOWSKI, T.B., PRESLER, P. \& ZUKOWSKY, C. 1983. Food habits of nototheniid fishes (Nototheniidae) in Admiralty Bay (King George Island, South Shetland Islands). Pol. Polar Res. 4(1):79-95.

MAIN, M.R., NICOL, G.J. \& ELDON, G.A. 1985. Distribution and biology of freshwater fishes in the Cook River to Paringa River area, South Westland. New Zealand Ministry of Agriculture and Fisheries, New Zealand. Fisheries environmental report 60.

MANTELATTO, F.L.M. 1995. Biologia reprodutiva de Canillectes ornatus Ordway, 1863 (Decapoda, Brachyura, Portunidae) na região de Ubatuba, SP, Brasil. Tese de doutorado. Universidade Estadual Paulista "Júlio de Mesquita Filho", Botucatu, SP.

MASUNARI, S. 1983. The Phytal of Amphiroa fragilissima (Linnaeus) Lamouroux, 1816. Stud. Neotrop. Fauna Environ. 18(3):151-161.

MCCORNICK, M.I. 1995. Fish feeding on mobile benthic invertebrates: influence of spatial variability in habitat associations. Mar. Biol. 121(4):627-638.

MEDEIROS, L.R.A. 1989. Meiofauna de praia arenosa da Ilha Anchieta, São Paulo. Tese de Mestrado. Universidade de São Paulo, São Paulo, SP.

MENDES, L.F. 2006. História natural dos amborés e peixes-macaco (Actinopterygii, Blennioidei, Gobioidei) do Parque Nacional Marinho do Arquipélago de Fernando de Noronha, sob um enfoque comportamental. Revta. bras. Zool. 23(3):817-823.

MENEZES, N.A. \& FIGUEIREDO, J.L. 1985. Manual de peixes marinhos do sudeste do Brasil. V. Teleostei (4). Museu de Zoologia da Universidade de São Paulo, São Paulo.

METRI, R. 2006. Ecologia de um banco de algas calcárias da reserva marinha biológica do Arvoredo, Sc, Brasil. Tese de doutorado. Universidade Federal do Paraná, Curitiba, PR.

MITTELBACH, G.G. \& PERSSON, L. 1998. The ontogeny of piscivory and its ecological consequences. Can. J. Fish. Aquat. Sci. 55(6):1454-1465.

MORING, J.R. 1979. Pacific coast interdial fishes. Sea Frontiers 25:22-30.

NAKAMURA, E. 1971. Food of two cohabiting tide-pool Cottidae. J. Fish. Res. Board. Can. 28(6):928-932.

NIEDER, J. 2001. Amphibious behaviour and feeding ecology of the four-eyed blenny Dialommus fuscus (Labrisomidae) in the intertidal zone of the island of Santa Cruz (Galapagos, Ecuador). J. Fish Biol. 58(3):755-767.

NORTON, T.A. \& BENSON, M.R. 1983. Ecological interactions between the brown seaweed Sargassum muticum and its associated fauna. Mar. Biol. 75(2):169-177.

PINNEGAR, J.K., TRENKEL, V.M., TIDD, A.N., DAWSON, W.A. \& DU BUIT, M.H. 2003. Does diet in Celtic Sea fishes reflect prey availability?. J. Fish Biol. 63(A):197-212.

PLATELL, M.E., POTTER, I.C. \& CLARKE, K.R. 1998. Do the habitats, mouth morphology and diets of the mullids Upeneichthys stotti and $U$. lineatus in coastal waters of south-western Australia differ? J. Fish Biol. 52(2):398-418

PRATCHETT, M.S. 2007. Dietary selection by coral-feeding butterflyfishes (Chaetodontidae) on the Great Barrier Reef, Australia. Raffles Bull. Zool. 14(3):171-176.

QUIJADA, P. \& CÁCERES, C. 2000. Patrones de abundancia, composición trófica y distribución espacial del ensamble de peces intermareales de la zona centro-sur de Chile. Rev. Chil. Hist. Nat. 73(4):739-747.

RANDALL, J.E. 1967. Food habits of reef fishes of the West Indies. Stud. Trop. Oceanogr. 5:665-847.

SANTOS, F.B. 2005. Utilização de micro-hábitats, ecologia trófica, ritmicidade e morfometria em peixes Blennioidei da região São Sebastião, São Paulo (Teleostei: Perciformes). Tese de doutorado. Universidade de São Paulo, São Paulo, SP.

SABINO, J. 1992. Partilha de recurso alimentar e espacial em duas espécies de emborês (Osteichthyes, Labrisomidae), em costões rochosos do litoral de Ubatuba, Estado de São Paulo. Dissertação de Mestrado. Universidade Estadual Paulista, São Paulo - SP, 81p.

SAZIMA, I. 1986. Similarities in feeding behavior between some marine and freshwater fishes in two tropical communities. J. Fish Biol. 29(1):53-65.

TANAKA, M.A. \& LEITE, F.P.P. 2003. Spatial scaling in the distribution of macrofauna associated with Sargassum stenophyllum (Mertens) Martius: analysis of faunal groups, gammarid life habits, and assemblage structure. J. Exp. Mar. Biol. Ecol. 293(1):1-22.

TARARAM, A.S. \& WAKABARA, Y. 1981. The mobile fauna - especially Gammaridea - of Sargassum cymosum. Mar. Ecol. Prog. Ser. 5:157-163.

ZAHORCSAK, P., SILVANO, R. \& SAZIMA, I. 2000. Feeding biology of a guild of benthivorous fishes in a sandy shore on south-eastern Brazilian coast. Revta. Bras. Biol. 60(3):511-518.

ZAMPROGNO, C. 1989. Distribuição e hábitos alimentares dos peixes na zona entremarés de recifes rochosos da Praia de Manguinhos, Espírito Santo. Tese de Mestrado. Universidade Estadual de Campinas, Campinas, SP.

ZAVALA-CAMIN, L.A. 1996. Introdução ao estudo sobre alimentação natural em peixes. EDUEM, Maringá.

Recebido em 07/03/08 Versão Reformulada recebida em 25/08/08 Publicado em 02/09/08 